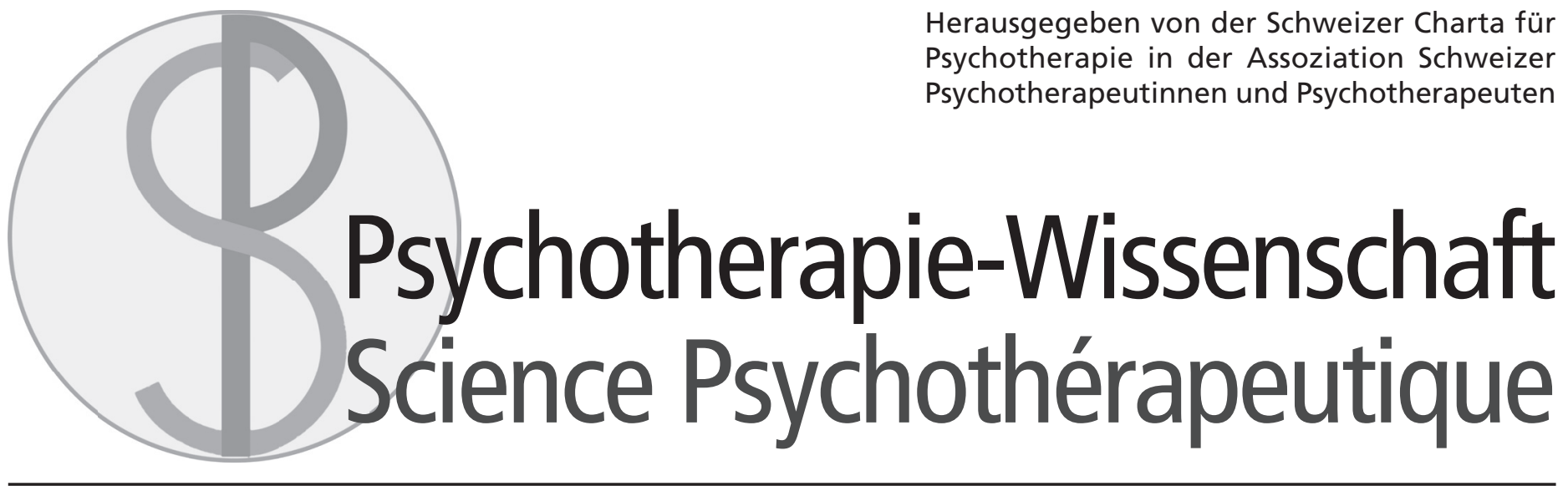

○ WWW.PSYCHOTHERAPIE-WISSENSCHAFT.INFO

\title{
Therapie depressiver Prozesse
}

Thérapie des processus dépressifs

10. Jahrgang

Heft 2 / 2020

Herausgegeben von Rosmarie Barwinski und Peter Schulthess

ISSN 1664-9583 


\section{Impressum}

Psychotherapie-Wissenschaft

ISSN 1664-9583 (Print-Version)

ISSN 1664-9591 (digitale Version)

10. Jahrgang Heft $2 / 2020$

https://doi.org/10.30820/1664-9583-2020-2

info@psychotherapie-wissenschaft.info

www.psychotherapie-wissenschaft.info

\section{Herausgeber}

Schweizer Charta für Psychotherapie in der Assoziation

Schweizer Psychotherapeutinnen und Psychotherapeuten

Geschäftsstelle ASP

Riedtlistr. 8

CH-8006 Zürich

Tel. +41432689300

www. psychotherapie.ch

\section{Redaktion}

Rosmarie Barwinski, Zürich

Nicola Gianinazzi, Lugano

Margit Koemeda, Zürich

Mario Schlegel, Zürich

Peter Schulthess, Zürich

Hinweise für AutorInnen befinden sich auf der Homepage der Zeitschrift: www.psychotherapie-wissenschaft.info

\section{Verlag}

Psychosozial-Verlag

Walltorstr. 10

D-35390 Gießen

+49642196997826

info@psychosozial-verlag.de

www.psychosozial-verlag.de

\author{
Abo-Verwaltung \\ Psychosozial-Verlag \\ bestellung@psychosozial-verlag.de
}

\section{Bezugsgebühren}

Jahresabonnement 44,90€ (zzgl. Versand)

Einzelheft $24,90 €$ (zzgl. Versand)

Studierende erhalten gegen Nachweis $25 \%$ Rabatt.

Das Abonnement verlängert sich um jeweils ein Jahr, sofern nicht eine Abbestellung bis acht Wochen vor Ende des Bezugszeitraums erfolgt.

ASP-Mitglieder wenden sich wegen des Abonnements bitte direkt an die ASP.

\section{Anzeigen}

Anfragen zu Anzeigen bitte an den Verlag:

anzeigen@psychosozial-verlag.de

Es gelten die Preise der auf www.psychosozial-verlag.de einsehbaren Mediadaten.

ASP-Mitglieder wenden sich bitte direkt an die Redaktion.

\section{Digitale Version}

Die Zeitschrift Psychotherapie-Wissenschaft ist auch online einsehbar: www.psychotherapie-wissenschaft.info

\section{(C) $(1) \Theta \Theta$}

Die Beiträge dieser Zeitschrift sind unter der Creative Commons Attribution-NonCommercial-NoDerivs 3.0 DE Lizenz lizensiert. Diese Lizenz erlaubt die private Nutzung und unveränderte Weitergabe, verbietet jedoch die Bearbeitung und kommerzielle Nutzung. Weitere Informationen finden Sie unter: creativecommons.org/licenses/by-nc-nd/3.0/de 
Editorial

Éditorial

Rosmarie Barwinski \& Peter Schulthess

\section{Titelthema:}

Therapie depressiver Prozesse

Thème principal:

Thérapie des processus dépressifs

Depression - eine Signatur unserer Zeit?

Erkenntnisse aus der LAC-Studie

La dépression - une caractéristique de notre temps ? 19

Conclusions de l'étude LAC

Marianne Leuzinger-Bobleber

Arbeitslosigkeitserfahrung, soziale Unterstützung und Depression

Ergebnisse der DEGS1-MH-Studie

Expérience du chômage,

de l'aide sociale et de la dépression

Résultats de l'étude DEGS1-MH

Stephan Müters, Lars E. Kroll,

Julia Thom \& Jens Hoebel

Der Gestalttherapeutische Ansatz

bei depressiven Erfahrungen

Gestalt Therapy Approach

to Depressive Experiences

Approche de Gestalt-thérapie

des expériences dépressives

Gianni Francesetti \& Jan Roubal

Depression - ein komplexes dynamisches System

La dépression - un système dynamique complexe

Günter Schiepek

Die Bedeutung der Elektrokonvulsionstherapie (EKT) in der multimodalen Behandlung

depressiver Störungen

L'importance de l'électroconvulsivothérapie (ECT)

dans le traitement multimodal des troublesdépressifs

Holger Himmighoffen \& Heinz Böker

Some contributions on the experience and therapy of depression published in the Italian journal

Psicoterapia e Scienze Umane

( «Psychotherapy and the Human Sciences»)

Alcune pubblicazioni sull'esperienza

e la psicoterapia della depressione apparse

sulla rivista Psicoterapia e Scienze Umane

Paolo Migone

74
5 Debatte

7 Débat

Antworten auf die Kommentare

von Greiner und Burda (Heft 2/2019)

81

Réponses aux commentaires de Greiner et Burda 84

Markus Erismann

\section{Originalarbeit \\ Article inédit}

Medium und Mediat in Psychotherapie

und Psychotherapiewissenschaft

Les médias et les médias dans la psychothérapie

et la science de la psychothérapie

Gerhard Burda

\section{Buchbesprechungen}

29 Amina Trevisan (2020).

Depression und Biographie. Krankheitserfahrungen migrierter Frauen in die Schweiz

Peter Schulthess

Tanja Krones \& Monika Obrist (Hrsg.). (2020).

31 Wie ich behandelt werden will.

Advanced Care Planning

39 Kathrin Schneider-Gurewitsch (2020).

Reden wir über das Sterben

46 Doris Signer-Brandan

Margit Koemeda (2019).

49 Tanzen vor Freude, Zittern vor Wut. Sich von

59 Gefühlen bewegen lassen. Ein Selbsthilfebuch mit körperpsychotherapeutischen Techniken

Peter Freudl

Tanja Lenz \& Ingrid Fleck (2020).

61 Exploratives Sandspiel. Wege zur Resilienz

Ursula Kübler

Kristina E. Schellinski (2020).

Individuation for Adult Replacement Children.

Ways of Coming into Being

Isabelle Meier

77 Kurt Greiner (2020).

Experimentelle Psychotherapiewissenschaft.

Das Methodenprogramm der Wiener

Therapieschulenforschung

Peter Schulthess 
Jürgen Kriz (2017).

Subjekt und Lebenswelt. Personzentrierte

Systemtheorie für Psychotherapie,

Beratung und Coaching

Peter Schulthess
Hermann Wegscheider (2020).

Dialog und Intersubjektivität in der Gestalttherapie. Von der jüdischen Tradition und Dialogphilosophie

$105 \mathrm{zu}$ relationalen Entwicklungen in der Psychoanalyse und Gestalttherapie

Peter Schulthess 


\section{Editorial}

Depressive Erkrankungen nehmen in den letzten Jahren weltweit zu. Die WHO geht davon aus, dass sie im Jahr 2020 die zweithäufigste Erkrankung darstellen. Galten sie bislang als gut behandelbar, zeigt sich nun, dass viele schwere Depressionen chronifizieren können und dass die Anzahl chronifizierter Depressionen steigt. Das war uns Grund genug, ein Heft zum Thema der Therapie depressiver Prozesse zu machen, in dem die Thematik vielseitig ausgeleuchtet wird, von aktueller Forschung bis hin zur Darstellung besonderer Ansätze der Therapie.

Marianne Leuzinger-Bobleber stellt die LAC-Studie vor (Langzeit-Studie chronischer Depressionen), in der Therapieverläufe psychoanalytischer Langzeitbehandlungen und solche mit Kognitiver Verhaltenstherapie untersucht und verglichen wurden. Beide Verfahren erwiesen sich als erfolgreich. Es liessen sich keine statistisch signifikanten Unterschiede zwischen den Verfahren finden bzgl. Symptomreduktion. Ebenso ergaben sich keine Unterschiede im Outcome, wenn man die Gruppe mit randomisierter Verteilung der PatientInnen gegen die Gruppe verglich, in der PatientInnen mit ihrer Wunschtherapie behandelt wurden. Dieses Ergebnis überrascht, da bisher die Kognitive Verhaltenstherapie psychoanalytischen Behandlungsmethoden als überlegen ausgewiesen wurde. Zudem konnte belegt werden, dass nach drei Jahren bei jenen PatientInnen die depressiven Symptome am deutlichsten zurückgingen, die die grössten strukturellen Veränderungen zeigten. Diese PatientInnen waren mit psychoanalytischen Methoden behandelt worden.

Stefan Müters, Lars E. Kroll, Julia Thom und Jens Hoebel untersuchten in der DEGS1_MH-Studie (Studie zur Gesundheit Erwachsener in Deutschland) die Bedeutung von sozialen Faktoren für die Entstehung und den Verlauf depressiver Krankheitsbilder. Konkret beschränkten sie sich auf die Wirkung von Arbeitslosigkeitserfahrung und sozialer Unterstützung im Hinblick auf depressive Erkrankungen. Frauen und Männer mit Arbeitslosigkeitserfahrung sind etwa doppelt so oft von Depressionen betroffen wie Erwerbstätige ohne Arbeitslosigkeitserfahrung in den letzten fünf Jahren. Die Analysen betonen die Bedeutung sozialer Ressourcen für den Zusammenhang zwischen Arbeitslosigkeit und Depressionen.

Gianni Francesetti und Jan Roubal beschreiben einen gestalttherapeutischen Ansatz zum Verständnis und zur Therapie depressiver Erfahrungen. Sie verfolgen einen radikalen dialogischen Ansatz und verstehen depressives Erleben als kokreiert in Beziehungen eines sozialen Systems. In der Therapiesituation zeigt sich, wie die Kommunikation zu depressivem Erleben führt, das beide erfasst, PatientIn und TherapeutIn. Im Besprechen dieses Phänomens bietet sich die Chance für die Therapie, das depressive Muster gemeinsam aufzubrechen. Dieser Beitrag wird im englischen Original wie auch in deutscher Übersetzung wiedergegeben.
Günter Schiepek beleuchtet die Ergebnisse verschiedener Depressionsstudien am Institut für Synergetik und Psychotherapieforschung in Salzburg. Im Gegensatz zum Ansatz mit manualisierten Therapievorgehen präferiert er eine personalisierte Psychotherapie. Er sieht depressive Prozesse als systemische Prozesse, zu denen sich in der Forschung bestimmte Muster abzeichnen. Bei Ordnungsübergängen zeigt sich eine kritische Instabilität, die prädikativ für einen besseren Therapieerfolg ist. Seine Forschungsgruppe hat eine spezielle App entwickelt, die tägliche Befindlichkeitsmessungen erlaubt. Neben klinischen Verbesserungen konnten auch Veränderungen der funktionellen Konnektivitätsdynamik neuronaler Netze aufgezeigt werden. Zum Schluss erläutert der Autor die Möglichkeit, nichtinvasive Stimulierungen der Gehirnaktivitäten mit Psychotherapie zu verbinden.

Holger Himmighoffen und Heinz Böker stellen die Elektrokonvulsionstherapie (EKT) als Teil einer multimodalen Behandlung depressiver Störungen vor. Das Verfahren war früher unter dem Namen Elektroschocktherapie bekannt und unter Verruf geraten wegen seiner schweren Nebenwirkungen. Die Autoren beschreiben die heutige EKT als ein Verfahren mit auf Dauer relativ wenig Nebenwirkungen, das seinen sinnvollen Platz bei chronifizierten Therapieverläufen hat, bei denen weder Psychotherapie noch Pharmakotherapie helfen. Dazu legen sie Forschungsresultate vor. EKZ ist keine Psychotherapie, sondern eine biologische, medizinische Therapie. Die Autoren zeigen, wie sich Psychotherapie und EKT ergänzen können. Die Redaktion publiziert diesen Beitrag, weil sie es als relevant erachtet, dass PsychotherapeutInnen davon wissen, dass die EKT in einigen Schweizer psychiatrischen Kliniken regelmässig praktiziert wird. Ausserhalb der Kliniken wird im öffentlichen Raum kaum darüber berichtet und diskutiert. Gern nehmen wir allfällige Lektürereaktionen und Diskussionsbeiträge in einem nächsten Heft auf, um eine Plattform für eine fundierte Debatte zur EKT anzubieten.

Den Abschluss des thematischen Teils bildet wie immer der Beitrag von Paolo Migone mit Literaturangaben und Abstracts aus der Zeitschrift Psicoterapia e Scienze Umane.

In Heft 2-2019 wurde ein Beitrag von Markus Erismann zum Wissenschaftsbegriff in der Psychotherapiewissenschaft von Kurt Greiner und Gerhard Burda kommentiert. Markus Erismann schrieb nun eine Entgegnung, die wir unter der Rubrik «Debatte» abdrucken. Diese Diskussion wurde von den damaligen HeftherausgeberInnen, Rosmarie Barwinski und Mario Schlegel, ausdrücklich gewünscht. Gern drucken wir auch weitere Diskussionsbeiträge aus der Leserschaft in künftigen Heften.

Zur Diskussion um die Psychotherapiewissenschaft passt auch der Beitrag von Gerhard Burda mit dem Titel «Medium und Mediat in Psychotherapie und Psychotherapiewissenschaft». Der Text vergleicht einen starren und einen 
dynamischen Zugang zur Wirklichkeit anhand der beiden Begriffe Mediat und Medium. Medien werden nicht als Wesen oder Identitäten, sondern als Selbst-Differenzen bzw. Verbindungs- und Trennungsverhältnisse in einem ontologischen Sinn aufgefasst. Unsere Wirklichkeit(en) kommen dieser medialistischen Auffassung nach dadurch zustande, dass selbst-differente Medien einander permanent mediatisieren. Beispiele aus der Psychotherapie verdeutlichen, wie der Veränderungsprozess Inter- und Intrapsychisches umgreift. Die Idee, dass der Medienbegriff als Klammer für sämtliche Psychotherapieformen dienen kann, wird in Richtung Psychotherapiewissenschaft weitergeführt.

Mehrere Buchbesprechungen runden dieses Heft ab. Wir wünschen eine anregende Lektüre!

Rosmarie Barwinski \& Peter Schulthess 


\section{Éditorial}

Les maladies dépressives ont augmenté dans le monde entier ces dernières années. L’OMS suppose qu'elles constitueront la deuxième maladie la plus répandue en 2020. Alors qu'elles étaient auparavant considérées comme facilement traitables, il devient maintenant évident que de nombreuses dépressions graves peuvent devenir chroniques et que le nombre de dépressions chroniques augmente. Ce fut une raison suffisante pour que nous produisions une brochure sur le thème de la thérapie de processus dépressifs, dans laquelle le sujet est éclairé de diverses manières, de la recherche actuelle à la présentation d'approches thérapeutiques spéciales.

Marianne Leuzinger-Bobleber présente l'étude LAC (étude à long terme des dépressions chroniques), dans laquelle les déroulements des traitements psychanalytiques à long terme et ceux de la thérapie cognitivocomportementale ont été examinés et comparés. Les deux méthodes se sont avérées efficaces. Aucune différence statistiquement significative entre les méthodes n'a pu être constatée en termes de réduction des symptômes. De même, il n'y a pas eu de différences de résultats lorsque l'on compare le groupe avec la distribution aléatoire des patients par rapport au groupe dans lequel ces derniers ont été traités avec la thérapie souhaitée. Ce résultat est surprenant, car la thérapie cognitivo-comportementale s'est avérée supérieure aux méthodes de traitement psychanalytique. Il a également été démontré qu'après trois ans, les symptômes dépressifs diminuaient de manière plus significative chez les patients qui présentaient les plus grands changements structurels. Ces patients avaient été traités avec des méthodes psychanalytiques.

Stefan Müters, Lars E. Kroll, Julia Thom et Jens Hoebel ont étudié, dans le cadre de l'étude DEGS1_MH (Étude sur la santé des adultes en Allemagne), l'importance des facteurs sociaux pour le développement et le déroulement des troubles dépressifs. Plus précisément, ils se sont limités à l'effet de l'expérience du chômage et du soutien social en ce qui concerne les troubles dépressifs. Les femmes et les hommes ayant une expérience du chômage sont environ deux fois plus susceptibles d'être touchés par la dépression que ceux qui ont occupé un emploi sans expérience du chômage au cours des cinq dernières années. Les analyses soulignent l'importance des ressources sociales dans la relation entre le chômage et la dépression.

Gianni Francesetti et Jan Roubal décrivent une approche thérapeutique de la Gestalt pour comprendre et traiter les expériences dépressives. Ils poursuivent une approche dialogique radicale et comprennent les expériences dépressives comme étant $c o$-créées dans les relations d'un système social. La situation thérapeutique montre comment la communication conduit à une expérience dépressive qui affecte à la fois le patient et le thérapeute. La discussion de ce phénomène offre la possibilité à la thérapie de rompre ensemble le schéma dépressif. Cet article est reproduit dans l'original anglais ainsi que dans la traduction allemande.
Günter Schiepek présente les résultats de diverses études sur la dépression menées à l'Institut de recherche sur la synergie et la psychothérapie de Salzbourg. Contrairement à l'approche des procédures de thérapie manuelle, il préfère une psychothérapie personnalisée. Il considère les processus dépressifs comme des processus systémiques pour lesquels certains modèles émergent dans la recherche. Les transitions d'ordre montrent une instabilité critique, qui est prédictive d'un meilleur succès thérapeutique. Son groupe de recherche a développé une application spéciale qui permet de mesurer quotidiennement l'état de santé. En plus des améliorations cliniques, l'existence de changements dans la dynamique de la connectivité fonctionnelle des réseaux neuronaux ont pu être démontrés. Enfin, l'auteur explique la possibilité de combiner la stimulation non invasive de l'activité cérébrale avec la psychothérapie.

Holger Himmighoffen et Heinz Böker présentent la électroconvulsivothérapie (ECT) dans le cadre d'un traitement multimodal des troubles dépressifs. Cette méthode était connue sous le nom de thérapie par électrochocs et a été discréditée en raison de ses graves effets secondaires. Les auteurs décrivent l'ECT actuelle comme une méthode ayant relativement peu d'effets secondaires à long terme, qui a sa place utile dans les processus thérapeutiques chroniques où ni la psychothérapie ni la pharmacothérapie ne peuvent aider. À cette fin, ils présentent des résultats de recherche. La TEC n'est pas une psychothérapie, mais une thérapie biologique et médicale. Les auteurs montrent comment la psychothérapie et l'ECT peuvent se compléter. La rédaction publie cet article parce qu'elle considère qu'il est pertinent que les psychothérapeutes sachent que l'ECT est régulièrement pratiquée dans certains hôpitaux psychiatriques suisses. En dehors des cliniques, il n'y a guère de rapports et de discussions publiques à ce sujet. Nous serons heureux d'inclure toute réaction et contribution à la discussion dans un prochain numéro afin de fournir une plateforme pour un débat bien fondé sur l'ECT.

Comme toujours, la section thématique se termine par une contribution de Paolo Migone avec des références et des résumés de la revue Psicoterapia e Scienze Umane.

Dans le numéro 2-2019, Kurt Greiner et Gerhard Burda ont commenté une contribution de Markus Erismann sur le concept de science dans la science de la psychothérapie. Markus Erismann a maintenant rédigé une réponse, que nous publions sous la rubrique "Débat ". Cette discussion a été expressément réclamée par les rédacteurs du magazine de l'époque, Rosmarie Barwinski et Mario Schlegel. Nous serions également heureux de publier d'autres contributions de nos lecteurs dans les prochains numéros.

La contribution de Gerhard Burda intitulée « média et médiat dans la psychothérapie et la science de la psychothérapie. " Le texte compare une approche rigide et dynamique de la réalité en utilisant les deux termes de 
médiat et média. Les médias ne sont pas compris comme des êtres ou des identités, mais comme des autodifférences ou des relations de connexion et de séparation dans un sens ontologique. Selon cette vision médialiste, notre (nos) réalité(s) provient (proviennent) du fait que les médias autodifférenciés se médiatisent en permanence les uns les autres. Des exemples de psychothérapie illustrent la manière dont le processus de changement englobe les aspects inter- et intrapsychiques. L'idée que le concept des médias peut servir de parenthèse pour toutes les formes de psychothérapie est poursuivie dans le sens de la science de la psychothérapie.

Plusieurs critiques de livres complètent ce numéro. Nous vous souhaitons une lecture inspirée!

Rosmarie Barwinski \& Peter Schulthess 


\section{Titelthema: Therapie depressiver Prozesse Thème principal: Thérapie des processus dépressifs}





\title{
Depression - eine Signatur unserer Zeit?'
}

\section{Erkenntnisse aus der LAC-Studie}

\author{
Marianne Leuzinger-Bobleber \\ Psychotherapie-Wissenschaft 10 (2) 11-18 2020 \\ www.psychotherapie-wissenschaft.info \\ CC BY-NC-ND \\ https://doi.org/10.30820/1664-9583-2020-2-11
}

\begin{abstract}
Zusammenfassung: Die LAC-Studie ist die wohl erste kontrollierte Psychotherapiestudie, die die Ergebnisse psychoanalytischer und kognitiv-verhaltenstherapeutischer Langzeitpsychotherapien bei chronisch Depressiven mit randomisierter und präferierter Zuweisung miteinander vergleicht. Beide Psychotherapieverfahren erwiesen sich als erfolgreich. Die Effektstärken und die vollständigen Remissionsraten waren höher als in anderen Studien. In zukünftigen Arbeiten werden nun Mediatoren- und Moderatorenvariablen untersucht, um beschreiben zu können, welche therapeutischen Faktoren in welchem der beiden Verfahren zu welchen Effekten geführt haben. In diesem Artikel werden die mittlerweile auch ausführlich publizierten Erkenntnisse aus der LAC-Studie sowie das methodische Vorgehen knapp skizziert.
\end{abstract}

Schlüsselwörter: chronische Depression, psychoanalytische und kognitiv-behaviorale Langzeittherapien, Outcomestudies, symptomatische und strukturelle Veränderungen

Depressionen haben in den letzten Jahrzehnten so stark zugenommen, dass sie nach Schätzungen der WHO im Jahr 2020 weltweit die zweithäufigste Krankheit sein werden. 20-30\% der Menschen mit einer depressiven Erkrankung entwickeln einen chronischen Verlauf. Chronifizierende Patient*innen zeigen besonders häufig sehr schwerwiegende Depressionen, viele depressive Episoden und psychiatrische und körperliche Komorbiditäten. Sie nehmen - verglichen mit anderen depressiven Patient*innen - länger Psychopharmaka und zeigen eine niedrigere Remissionsrate. Chronische Depressionen sind zudem mit enormem Leid für die Betroffenen und ihre Familien sowie mit hohen direkten und indirekten Gesundheitskosten verbunden.

Lange Zeit galt die Depression als eine Erkrankung mit einer relativ guten Behandlungsprognose, doch dies hat sich in den letzten Jahrzehnten geändert. Ergebnisse aus der epidemiologischen Forschung zeigten, dass Depressionen häufig eine wiederkehrende Erkrankung mit einer hohen Rückfallquote sind und chronisch werden. $50 \%$ der depressiven Patient*innen erleiden nach der ersten depressiven Episode ein Rezidiv, 70 \% nach der zweiten und $90 \%$ nach der dritten Episode. Darüber hinaus haben sich pharmakologische und kognitiv-verhaltenstherapeutische als auch psychodynamische Kurztherapien als weit weniger erfolgreich erwiesen als erhofft: $50 \%$ aller depressiven Patient*innen erleiden nach jeder Form von Kurzpsychotherapie einen Rückfall. 20-30\% aller depressiven Patient*innen sprechen nicht positiv auf Medikamente an. Von denjenigen, die das doch tun, erleidet ein Drittel

1 Dieser Beitrag basiert auf früheren Arbeiten (Leuzinger-Bohleber et al., 2019a, c, 2020; Kaufhold et al., 2019), in denen sich weiterführende Literaturangaben befinden. innerhalb eines Jahres einen Rückfall, $75 \%$ innerhalb von fünf Jahren. Für diese Patient*innen können langfristige psychoanalytische Therapien oder Psychoanalysen eine Alternative bieten. In der repräsentativen DPV-OutcomeStudie haben zum Beispiel rund $80 \%$ aller 402 ehemaligen Psychoanalyse-Patient*innen mit psychoanalytischen Langzeittherapien eine nachhaltige Verbesserung ihrer psychopathologischen Symptome sowie ihrer Objektbeziehungen, ihrer beruflichen Entfaltungen und ihrer allgemeinen Lebensqualität gezeigt. Unter ihnen waren $27 \%$, die als depressiv diagnostiziert worden waren, meist in Kombination mit einigen Persönlichkeitsstörungen. Als unerwartetes Ergebnis der Studie zeigte sich die enge Verbindung zwischen Trauma und schwerer psychischer Erkrankung: $62 \%$ der Patient*innen waren schwer traumatisierte Kinder aus dem Zweiten Weltkrieg (Leuzinger-Bohleber et al., 2003).

Nach Bohleber (2005) ist die Depression in den Sozialwissenschaften zu einer Signatur unserer Zeit avanciert, in der sich traditionelle Strukturen und klare Verhaltenserwartungen weitgehend aufgelöst haben. Abgrenzungsphänomene und der enorme Zuwachs an individuellen Wahlmöglichkeiten von Lebensperspektiven führen zu einem Verlust an sozialer Sicherheit und machen die eigene Identität zum Lebensprojekt des Individuums. Der französische Soziologe Ehrenberg (2016) erklärt in seiner Studie das erschöpfte Selbst zur Krankheit der heutigen Gesellschaft, deren Verhaltensnormen nicht mehr auf Schuld und Disziplin, sondern vor allem auf Verantwortung und Initiative beruhen. An die Stelle des spätbürgerlichen Individuums scheint ein Individuum zu treten, das die Idee hat, dass «alles möglich ist», und das von der Angst um seine Selbstverwirklichung geprägt ist, die sich leicht bis zum Gefühl der Erschöpfung steigern 
kann. Der Druck zur Individualisierung spiegelt sich in Versagens-, Scham- und Unzulänglichkeitsgefühlen und schliesslich in depressiven Symptomen wider. Die «klassische Neurose» ist für Ehrenberg eine Krankheit des Individuums, die durch Konflikte zwischen Erlaubtem und Verbotenem determiniert wird, während die Depression für ihn als Krankheit des Individuums gesehen wird, die durch die Spannung zwischen Möglichem und Unmöglichem entsteht. Die Depression wird so zu einer Tragödie der Unzulänglichkeit.

Solche sozialwissenschaftlichen und historischen Analysen fordern auch die Psychoanalyse heraus, das Thema Depression neu zu untersuchen und den Stand ihrer Forschung neu zu bewerten. Inzwischen sind die Ergebnisse psychoanalytischer Kurzzeittherapien, die den Kriterien der evidenzbasierten Medizin entsprechen, durch viele Studien bestätigt worden. Demgegenüber liegen nach wie vor nur wenige Studien zu den Ergebnissen von Langzeitpsychotherapien und Psychoanalysen vor. Daher beschloss 2005 eine multizentrische Forschungsgruppe von Psychoanalytiker*innen und kognitiven Verhaltenstherapeut*innen, eine vergleichende Psychotherapiestudie zu den Ergebnissen kognitiv-verhaltenstherapeutischer und psychoanalytischer Langzeitbehandlungen $\mathrm{zu}$ initiieren, die sogenannte LAC-Studie (Projektleitung: M. Leuzinger-Bohleber, M. Hautzinger, M. Beutel, G. Fiedler, W. Keller). Wir konzipierten die Studie in enger Zusammenarbeit mit der Forschungsgruppe von Phil Richardson, Peter Fonagy und David Taylor aus London, die damals ebenfalls eine Studie über die Ergebnisse psychoanalytischer Langzeitpsychotherapien bei schwer zu behandelnden Depressionen plante, die Tavistock-Depressionsstudie. Inzwischen sind sowohl die Ergebnisse der Tavistock- (Fonagy et al., 2015) als auch der LAC-Studie (Leuzinger-Bohleber et al., 2019a, c) veröffentlicht worden. Diese und mehrere andere Studien zeigen die positiven Ergebnisse von psychoanalytischen (PAT) und verhaltenstherapeutischen (KVT) Langzeittherapien für depressive Patient*innen auf.

\section{Die LAC-Studie}

Die LAC-Studie (Langzeitbehandlungen chronisch Depressiver) ist eine randomisiert kontrollierte Studie zum Vergleich der Ergebnisse kognitiv-behavioraler und psychoanalytischer Langzeitbehandlungen chronisch depressiver Patient*innen mit randomisierter und präferierter Zuweisung. Die Neugierde, mehr über die Faktoren zu erfahren, die bei psychoanalytischen Behandlungen im Vergleich zu längeren kognitiv-behavioralen Therapien eine Rolle spielen, die drohende gesellschaftliche Marginalisierung psychoanalytischer Verfahren, aber auch das Interesse einiger KVT-Forscher*innen an einer längeren, von den Krankenkassen finanzierten Behandlung, waren einige der Gründe für diese multizentrische Studie zu den Ergebnisse von kognitiv-behavioralen und psychoanalytischen Langzeitbehandlung bei chronisch depressiven Patient*innen.
Die Planung und Durchführung der LAC-Studie fand vor dem Hintergrund vieler kontroverser Diskussionen statt (s.z. B. Leuzinger-Bohleber et al., 2020). So entschied sich die Forschungsgruppe beispielsweise für ein Design, das eine naturalistische Studie mit einer experimentellen kombiniert. Im Gegensatz zu vielen Studien der vergleichenden Psychotherapieforschung, in denen aus methodischen und pragmatischen Gründen fortgeschrittene Psychologiestudierende Personen mit genau definierten Symptomen (oft ebenfalls Studierende) nach einer manualisierten Therapiemethode behandelten, wurden in der LAC-Studie chronisch depressive Patient*innen interviewt, wie sie heute in Deutschland in Privatpraxen von Psychotherapeut*innen in Langzeitpsychotherapien behandelt werden. Wir erwarteten, dass viele dieser Patient*innen bereits mehrere Kurztherapien mit nur begrenztem Erfolg oder sogar negativem Ergebnis durchlaufen hatten und deshalb ein bestimmtes therapeutisches Verfahren bevorzugten. Wir vermuteten, dass sie daher nicht bereit wären, sich für eine Langzeitbehandlung randomisieren zu lassen. Deshalb konnten sie in der LAC-Studie zwischen den beiden Therapien KVT und PAT wählen. ${ }^{2}$ Wenn sie keine klare Präferenz hatten und dazu bereit waren, wurden sie randomisiert.

Randomisierung der Patient*innen, genau beschriebene Einschlusskriterien, bezogen auf die Verfahren verblindete Rater, reliable Messinstrumente, manualisierte und auf ihre Adhärenz überprüfte Therapieverfahren sowie die genaue Beschreibung der Stichproben, der drop-outs und der angewandten statistischen Verfahren etc. gehören zu den Kriterien der sogenannten evidencebased-medicine. Diese Kriterien müssen erfüllt sein, damit Studien sowohl in der Welt der Psychotherapieforschung als auch der Gesundheitssysteme anerkannt werden. Daher versuchte die Forschungsgruppe der LAC-Studie allen diesen Kriterien gerecht zu werden.

Darüber hinaus wurden die bekannten wissenschaftstheoretischen und methodischen Bedenken der psychoanalytischen Community ernst genommen und eine multiperspektivische Annäherung an den Therapieerfolg bei diesen difficult-to-treat-Patient*innen gewählt. So wird in der zweiten Hauptpublikation der LAC-Studie ein Aspekt dieser komplexen Problematik exemplarisch zur Diskussion gestellt: In der Welt der evidence-based-medicine werden bekanntlich fast ausschliesslich Symptomveränderungen als Erfolg für Psychotherapien betrachtet, während nach psychodynamischer Auffassung vor allem Veränderungen der inneren Objektwelt, sogenannte Strukturveränderungen, erfolgreiche Psychotherapien charakterisieren, denn das Ziel psychoanalytischer Behandlungen geht über Symptomveränderungen weit hinaus und wird, wie schon Freud dies postulierte, beschrieben als die Fähigkeit zu lieben, zu arbeiten und das Leben zu geniessen. Daher wurden in der LAC-Studie symptomatische und strukturelle Veränderungen miteinander in Beziehung gesetzt.

22005 waren PAT und KVT die einzigen von Krankenversicherungen in Deutschland - aufgrund ausreichender evidenzbasierter Studien zu ihrer Wirksamkeit -anerkannten Psychotherapien. 